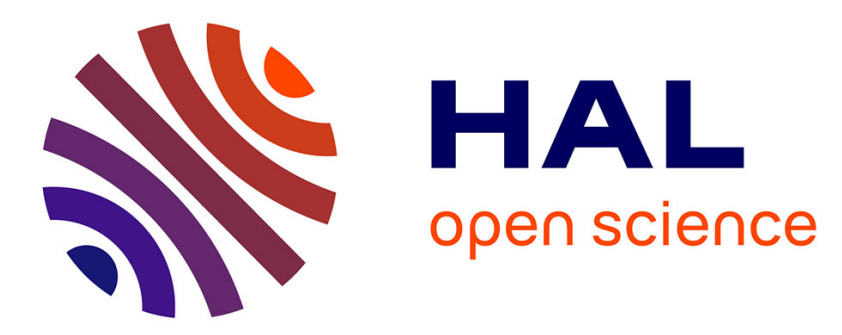

\title{
L'impact de la relation prédécesseur/ successeur sur le déroulement du processus de succession dans les entreprises familiales
}

\author{
Alain Fayolle, Salma Fattoum
}

\section{- To cite this version:}

Alain Fayolle, Salma Fattoum. L'impact de la relation prédécesseur/ successeur sur le déroulement du processus de succession dans les entreprises familiales. La Revue des Sciences de Gestion, 2008. hal-01892888

\section{HAL Id: hal-01892888 \\ https://hal.science/hal-01892888}

Submitted on 10 Oct 2018

HAL is a multi-disciplinary open access archive for the deposit and dissemination of scientific research documents, whether they are published or not. The documents may come from teaching and research institutions in France or abroad, or from public or private research centers.
L'archive ouverte pluridisciplinaire HAL, est destinée au dépôt et à la diffusion de documents scientifiques de niveau recherche, publiés ou non, émanant des établissements d'enseignement et de recherche français ou étrangers, des laboratoires publics ou privés. 


\section{L’impact de la relation prédécesseur/ successeur sur le déroulement du processus de succession dans les entreprises familiales}

Salma Fattoum

Doctorante

Dynamiques des processus entrepreneuriaux

EM Lyon

\section{fattoum@em-lyon.com}

\section{Résumé}

Une étude menée par l'Ifop en septembre 2003 auprès de dirigeants de petites et moyennes entreprises (PME) pour le compte du conseil supérieur de l'ordre des experts-comptables, l'agence pour la création d'entreprise et l'assemblée des chambres françaises de commerce et d'industrie, fait ressortir que parmi les 843 personnes interrogées, 76\% sont des futurs cédants et $24 \%$ ont l'intention de transmettre leurs entreprises dans les cinq prochaines années. L'entreprise familiale qui intègre ces statistiques se trouve particulièrement fragilisée lors du passage d'une génération à l'autre. Elle est non seulement exposée aux menaces qui guettent tout type d'entreprise mais elle doit encore affronter des dangers propres à sa nature familiale. Le caractère familial concerne des entreprises dont le poids sur l'activité économique des nations est loin d'être négligeable aussi bien pour les pays développés que pour ceux en voie de développement.

En Tunisie, l'impulsion pour l'entreprise privée a été donnée dans les années 60. Presque un demi-siècle plus tard, ces entrepreneurs qui ont bénéficié de l'aide de l'Etat pour s'installer, arrivent aujourd'hui à l'âge de la retraite et se trouvent confrontés à une problématique de taille, celle de leur départ et de la mise en place effective d'une relève pouvant assurer la pérennité et le développement de leurs entreprises. Une étude effectuée par Morris et al. (1997) a montré qu'un des principaux facteurs de succès du processus de succession découle de la nature de la relation qui existe parmi tous les membres de la famille.

Notre objectif, dans ce papier, est de comprendre la nature de la relation prédécesseur/successeur durant le processus de succession dans les entreprises familiales tunisiennes en mettant l'accent sur les réflexions à mener pour réussir ce changement générationnel.

Mots clés : entreprise familiale, succession, prédécesseur, successeur, désengagement. 


\section{L’impact de la relation prédécesseur/ successeur sur le déroulement du processus de succession dans les entreprises familiales}

\section{Résumé}

Une étude menée par l'Ifop en septembre 2003 auprès de dirigeants de petites et moyennes entreprises (PME) pour le compte du conseil supérieur de l'ordre des experts-comptables, l'agence pour la création d'entreprise et l'assemblée des chambres françaises de commerce et d'industrie, fait ressortir que parmi les 843 personnes interrogées, 76\% sont des futurs cédants et $24 \%$ ont 1 'intention de transmettre leurs entreprises dans les cinq prochaines années. L'entreprise familiale qui intègre ces statistiques se trouve particulièrement fragilisée lors du passage d'une génération à l'autre, l'entreprise familiale se trouve particulièrement fragilisée. Elle est non seulement exposée aux menaces qui guettent tout type d'entreprise mais elle doit encore affronter des dangers propres à sa nature familiale. Le caractère familial concerne des entreprises dont le poids sur l'activité économique des nations est loin d'être négligeable aussi bien pour les pays développés que pour ceux en voie de développement.

En Tunisie, l'impulsion pour l'entreprise privée a été donnée dans les années 60. Presque un demi-siècle plus tard, ces entrepreneurs qui ont bénéficié de l'aide de l'Etat pour s'installer, arrivent aujourd'hui à l'âge de la retraite et se trouvent confrontés à une problématique de taille, celle de leur départ et de la mise en place effective d'une relève pouvant assurer la pérennité et le développement de leurs entreprises. Une étude effectuée par Morris et al. (1997) a montré qu'un des principaux facteurs de succès du processus de succession découle de la nature de la relation qui existe parmi tous les membres de la famille.

Notre objectif, dans ce papier, est de comprendre la nature de la relation prédécesseur/successeur durant le processus de succession dans les entreprises familiales tunisiennes en mettant l'accent sur les réflexions à mener pour réussir ce changement générationnel.

Mots clés : entreprise familiale, succession, prédécesseur, successeur, désengagement. 


\section{Introduction}

La globalisation des marchés oblige désormais les entreprises, qu'elles soient grandes ou petites, à s'adapter et à se transformer pour répondre à des situations et des exigences nouvelles. L'entreprise familiale qui représente une forme d'organisation ancienne et très répandue dans le monde (Gersick et al., 1997) n'échappe pas à cette réalité. Ce type d'organisation concerne des entreprises dont le poids sur l'activité économique des nations est loin d'être négligeable aussi bien pour les pays développés que pour ceux en voie de développement. Il ressort par exemple d'une étude effectuée par Allouche et Amann (2003) que $90 \%$ des entreprises aux Etats-Unis sont possédées par des familles et qu'elles contribuent à réaliser entre 30 et $60 \%$ du PNB; en Europe Occidentale, entre 45 et $65 \%$ du PNB (et de l'emploi) est assuré par des entreprises familiales.

Toutefois, lors du passage d'une génération à l'autre, l'entreprise familiale se trouve particulièrement fragilisée. Elle est non seulement exposée aux menaces qui guettent tout type d'entreprise mais elle doit encore affronter des dangers propres à sa nature familiale. D'après une étude américaine effectuée en 1973 (Cadieux et Lorrain, 2002), seulement 30\% des entreprises familiales survivent à leur transfert à la seconde génération et moins de $10 \%$ survivent à un tel transfert à la troisième génération. Considérant en outre le nombre d'années nécessaires pour la préparation de ce passage dans de bonnes conditions (Haddadj, 1998), cela nous donne une idée sur l'importance de la succession et les problèmes qu'elle peut susciter.

Plusieurs études américaines et européennes se sont intéressées à la succession dans les entreprises familiales. Cependant, ce sujet demeure encore peu étudié dans les pays en voie de développement et plus particulièrement en Afrique où cette forme est de loin la plus répandue (Tchankam, 1998). En Tunisie, l'impulsion pour l'entreprise privée a été donnée dans les années 60. Presque un demi-siècle plus tard, ces entrepreneurs qui ont bénéficié de l'aide de l'Etat pour s'installer, arrivent aujourd'hui à l'âge de la retraite. Comment se présente alors leur départ? La succession est-elle "assurée"? Si on ne peut spéculer sur la réussite de cette succession, peut-on au moins décrire les phases de celle-ci dans un premier temps et réfléchir aux conditions qui pourraient l'affecter ? Malgré leur centralité pour l'avenir du tissu économique tunisien, ces questions attendent encore un éclairage académique. A partir de ce constat, nous avons cherché à nous intéresser aux deux questions suivantes : Comment se déroule le processus de succession (père- enfant) dans les entreprises familiales tunisiennes et 
comment la relation prédécesseur/ successeur peut-elle constituer un facteur de succès ou d'échec pour la relève?

Notre objectif est donc de mieux saisir la nature de la relation prédécesseur/successeur durant le processus de succession dans les entreprises familiales tunisiennes en mettant l'accent sur les réflexions à mener pour réussir ce changement générationnel. Une première section propose une analyse de la littérature sur l'entreprise familiale et le processus de succession. Ensuite, nous présentons dans une deuxième section, les aspects méthodologiques de notre étude réalisée auprès de six entreprises familiales tunisiennes qui ont été observées à différents stades du processus de succession. La présentation et la discussion des résultats font l'objet d'une troisième section.

\section{Entreprise familiale et changement générationnel}

Nombreux sont les auteurs qui se sont intéressés à la définition de l'entreprise familiale. Amann (1999) en a fait une synthèse. Il en ressort que le contrôle du capital par la famille constitue un point important qu'on retrouve dans plusieurs définitions (Gallo et Estapé, 1992 ; Barnes et Hershon, 1976 ; Handler, 1989 ; Hollander et Elman, 1988). Il y a en plus la participation de la famille dans la gestion de l'entreprise (Hugron, 1993 ; Cadieux, 1999) et aussi les liens étroits entre l'entreprise et la famille (Davis et Tagiuri, 1982).

Dans le cadre de notre travail, nous nous référons à la définition donnée par Cadieux (1999, p. 11), qui qualifie de familiale toute entreprise « sous contrôle individuel ou familial dont la majorité des dirigeants - il peut n'y en avoir qu'un - sont les membres de la même famille, qu'elle soit nucléaire ou élargie, que ces derniers y contrôlent la gestion effective et que ceuxci ont une volonté ferme de transmettre l'entreprise à la génération suivante ».

Une étude effectuée par Morris et al. (1997) fait ressortir que la qualité des relations qui existent entre les membres de la famille peut constituer une source potentielle d'avantage compétitif. Ainsi, il est important que les prédécesseurs veillent à ce qu'il y ait un esprit d'équipe parmi tous les membres de leur famille.

Après avoir défini ce que nous entendons par « entreprise familiale » en mettant en liaison les deux principaux sous-systèmes qui s'y rattachent, à savoir la famille et l'entreprise, nous allons nous intéresser dans ce qui suit au concept même de succession à travers l'étude des étapes de ce processus en privilégiant l'approche processus.

\subsection{Le processus de succession}

Dans la littérature disponible sur la succession dans les entreprises familiales, deux types de processus de succession sont identifiables : celui de la propriété et celui de la direction 
(Churchill et Hatten, 1987 ; Hugron, 1993 ; Morris et al., 1997). Les deux processus comportent chacun quatre étapes distinctes. Pour le premier, il s'agit de l'amorce de la décision, la décision elle-même, la consultation auprès d'experts et la finalisation du transfert de propriété. Pour le second, c'est l'incubation, le choix du successeur, le règne conjoint et le désengagement (Hugron et Dumas, 1993). Cadieux et Lorrain (2002) ont effectué une synthèse des recherches qui se sont intéressées à la compréhension du processus de succession et ont identifié, notamment, les travaux de Barnes et Hershon, 1976; Churchill et Hatten, 1987; Handler, 1989; Hugron et Dumas, 1993; Gersick et al.,1997. Il en ressort que le premier type de processus (propriété) décrit l'évolution de l'entreprise en général, tandis que le second (direction) s'intéresse plus particulièrement à l'évolution et l'implication des principaux acteurs que sont le prédécesseur et le successeur. Notre recherche s'inscrivant plutôt dans le deuxième type de processus, notre travail s'intéressera, par conséquent, principalement au processus de transfert de la direction.

Dans la première étape qui est celle de l'incubation, le prédécesseur s'intéresse à la gestion des différentes responsabilités dans son entreprise et bien qu'il ait l'intention de céder un jour à ses enfants l'entreprise qu'il a fondée, il y a peu de chances que le successeur soit impliqué dans l'organisation durant cette étape du processus. Dans la deuxième étape, il s'agit de l'intégration du successeur. Ce dernier doit disposer des capacités entrepreneuriales et de gestion suffisantes pour pouvoir assurer la continuité et le développement des activités (Bayad et Barbot, 2002). La troisième étape du processus, le règne conjoint, se caractérise par l'entrée officielle du successeur à ce titre dans l'entreprise (Stravou, 1998) ${ }^{1}$.

Il est à souligner que le règne conjoint peut créer certaines tensions au sein de l'entreprise entre les deux acteurs ce qui n'est pas sans conséquences sur l'activité de l'entreprise familiale. Enfin, la dernière étape, le désengagement, est caractérisée par le retrait du prédécesseur des différentes responsabilités inhérentes à l'entreprise. Nous analysons dans ce qui suit, plus en profondeur, le désengagement du prédécesseur à travers les notions de perte d'identité et de pouvoir.

\subsection{Le prédécesseur face au désengagement}

Les dirigeants d'entreprises familiales sont le plus souvent très attachés à leurs entreprises, ils s'y investissent totalement (Baumert, 1992). De ce fait, le détachement de leurs entreprises constitue une décision et un acte difficiles. Certains éprouvent d'ailleurs lors de la

\footnotetext{
${ }^{1}$ Cité dans Cadieux L., Lorrain J., et Hugron P. (2000), p.6
} 
transmission de leur entreprise, une sorte de perte d'identité et de pouvoir, ils se sentent inutiles et refusent de faire face à ce sentiment qui s'accentue au fur et à mesure que leur désengagement devient effectif. En effet, le chef d'entreprise perçoit souvent son entreprise comme une véritable prolongation de sa personnalité et s'identifie totalement à elle (Catry et Buff, 1996; Duchéneaut, 1996). De ce fait, transmettre son entreprise revient pour le prédécesseur à perdre une partie de lui-même à travers la rupture du lien fortement investi qu'il a envers son entreprise (Pailot, 1998). L'auteur mentionne que le dirigeant refuse consciemment ou inconsciemment d'abandonner un objet d'attachement central dans la construction de son identité personnelle et sociale à savoir son entreprise. De surcroît quitter l'entreprise s'accompagne d'un amoindrissement de la reconnaissance sociale (Pailot, 1998) et signifie souvent pour lui de ne plus servir à rien ou encore d'accepter une mort symbolique et parfois physique.

Le prédécesseur qui a été pendant toute sa vie professionnelle un homme de pouvoir (Baumert, 1992) a du mal à renoncer à ce pouvoir et cherche plutôt à le conserver voire à le consolider. Les chefs d'entreprise qui sont confrontés à ces situations se posent beaucoup de questions « comment vais-je pouvoir continuer à vivre, à exister, à rester valable, à avoir une image positive de moi-même si je me sépare de mon entreprise? ». Certains y répondent facilement dès le développement de leur entreprise en déléguant un maximum de responsabilités (Bruneau, 2002) et en transmettant leur savoir-faire. D'autres dirigeants ont du mal à affronter cette situation car dès le départ ils ne s'y sont pas vraiment préparés : paternalisme et pouvoir centré sur le chef, prévalence de l'affectif et des considérations familiales (Bruneau, 2002). Le dirigeant refuse d'affronter cette perte du pouvoir qui devient inévitable une fois la transmission effectuée et il fait le deuil de son entreprise. Selon Pailot (1998), la théorie du deuil est une théorie de la perte d'un lien d'attachement. Suite à des entretiens avec des dirigeants cédants, Bruneau (2002) mentionne que la transmission de l'entreprise pour son dirigeant est comparable à la perte d'un jouet pour l'enfant. En effet, l'enfant tient énormément à ses jouets, il y a un lien affectif qui s'est crée entre eux. Qu'arrivet-il alors si jamais il devait s'en séparer : un processus de deuil. Ce travail de deuil est d'ailleurs bénéfique pour la personne en question car il lui permet d'extérioriser ses affects sur de nouveaux objets. Bien que la succession soit assurée par leurs enfants, les dirigeants ont l'impression d'avoir perdu leurs entreprises en les transmettant, ils ont aussi peur que les successeurs rejettent ce qui a été accompli (Kets de Vries, 1998). Nous allons nous intéresser maintenant au comportement du successeur face à ses nouvelles responsabilités de dirigeant. 


\subsection{Le successeur et son nouveau rôle de dirigeant}

Il convient de souligner qu'il faut en moyenne entre deux et quatre ans pour que le successeur développe une image de crédibilité auprès de ses pairs (Barach et al., 1988) ${ }^{2}$ et pour qu'il soit accepté par le personnel et l'organisation. Ces auteurs énumèrent quatre dimensions facilitant l'intégration du successeur qui sont l'acceptabilité (partager la culture et connaître le métier de l'entreprise familiale), la crédibilité (avoir des compétences reconnues pour diriger l'entreprise familiale), la légitimité (avoir confiance en soi et la confiance des autres), et enfin le leadership (avoir une vision stratégique et une capacité à atteindre les objectifs). Le successeur doit aussi montrer son engagement, son attachement et son implication envers l'entreprise. Des études basées sur les perceptions du successeur (Barach et al., 1988), se sont intéressées en partie à ce que ces nouveaux dirigeants ressentent après avoir intégré l'entreprise familiale. Les résultats montrent que les successeurs se sentent observés par tous, ils disent qu'ils n'ont pas le droit à l'erreur. Certains successeurs apprécient le fait que le prédécesseur ne quitte pas l'entreprise trop rapidement, ceci les met en confiance et fonctionne comme un «outil de motivation pour les équipes et les interlocuteurs extérieurs » (Bruneau, 2002). D'autres en revanche n'acceptent pas la présence continuelle du prédécesseur au quotidien. La relation entre le prédécesseur et le successeur apparaît alors comme «l'accord réciproque sur une place à faire à l'autre» (Bruneau, 2002). Cette relation dépend en partie du vécu du successeur, de sa personnalité et de ses expériences.

Dans son ouvrage «L'identité au travail», Renaud Sainsaulieu (1985) montre le rôle de l'entreprise et des rapports de travail dans la construction de l'identité. Tout au long de sa vie, le dirigeant construit son identité personnelle et professionnelle à travers les différents évènements externes et les évolutions internes par lesquelles il passe. Dans le contexte de l'entreprise familiale, Carter et McGoldrick (1988) mentionnent que l'évolution du successeur dépend d'abord de lui, ensuite de sa capacité à développer sa propre identité. C'est à travers la confiance qui lui a été faite par les différents membres de l'entreprise et de la famille, l'écoute qui lui a été donnée et le sentiment de sa valeur, que le successeur apprend à avoir confiance en lui-même, à prendre des risques et à affronter des situations nouvelles qui vont contribuer à façonner son identité et à construire une certaine « image de soi » (Roussillon, 1998).

Haddadj et D'Andria (2001) mentionnent que le dirigeant n'arrive jamais dans l'entreprise avec des compétences managériales innées. C'est en effet à travers un processus de socialisation et d'interactions avec les personnes présentes que le successeur s'expose aux

\footnotetext{
${ }^{2}$ Cité dans Bayad M. et Barbot M-C. (2002), p. 5
} 
exigences requises par l'entreprise (Fiegener et al., 1996). C'est aussi à travers son expérience dans l'entreprise familiale que le successeur acquiert des connaissances, des valeurs propres à la famille, une culture, une identité et un statut. Il est primordial que dès son entrée dans l'entreprise une responsabilité précise lui soit confiée. En effet, seuls le rôle et la responsabilité transforment le spectateur en acteur (Meister, 1999). D'après une étude de Boiteux et Hugron (1998), la seule formation que le prédécesseur valorise est celle qui permettrait de réduire la durée de l'expérience de son successeur.

\subsection{Une synthèse sous une forme d'un questionnement}

Cette analyse de la littérature nous a permis d'avoir une idée plus claire sur les points critiques de la succession en entreprise familiale à travers les aspects auxquels nous nous sommes intéressés qui sont liés principalement au prédécesseur et au successeur. Après avoir présenté le déroulement des différentes étapes du processus de succession, nous avons tenté de décrire celles qui concernent le prédécesseur lorsqu'il se rapproche du moment où il devrait se retirer de l'entreprise familiale. Se trouvant confronté à une situation nouvelle pour lui, le prédécesseur ressent à la fois une sorte de perte d'identité et de pouvoir. Se dégager de l'entreprise qu'il a -dans la majorité des cas- fondée, signifie pour lui se retirer de ce qui avait donné un sens à toute son existence et accepter de faire le deuil de cette «perte ». Dans un second temps, nous nous sommes intéressés au comportement du deuxième acteur principal de la succession à savoir l'engagement du successeur et l'endossement d'un nouveau rôle de dirigeant. La figure 1 tente de représenter le processus de succession qui intervient sous la double influence des interactions qu'il entretient avec la famille et l'environnement de l'entreprise.

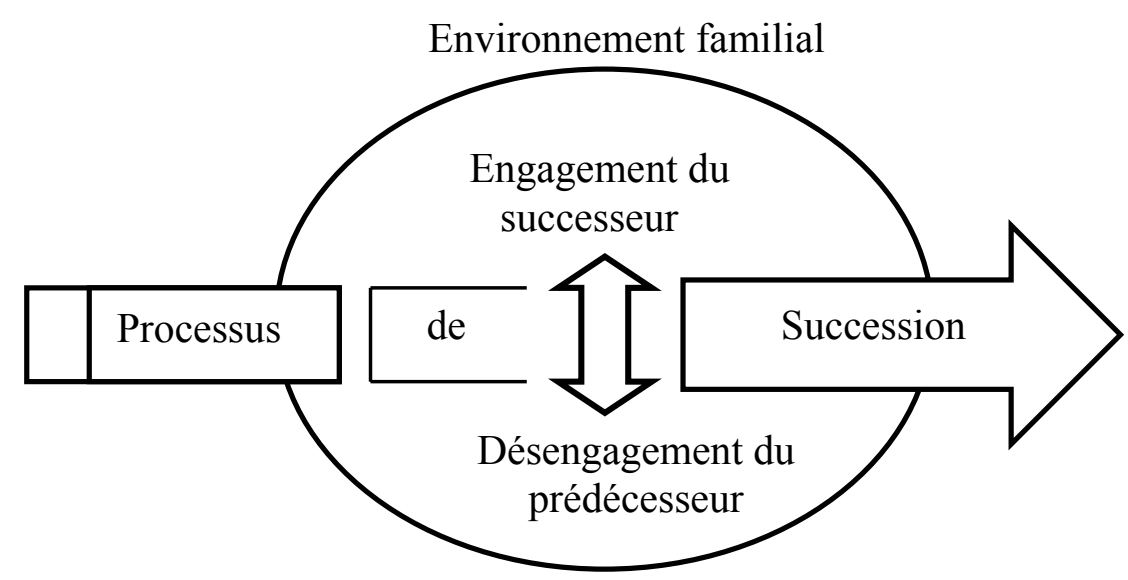

Environnement de l'entreprise

Figure 1. Une représentation du processus de succession de l'entreprise familiale 
La littérature concernant les entreprises familiales et le processus de succession a suscité de nombreuses questions. En effet, nous nous demandons comment ce processus est vécu concrètement par les entreprises tunisiennes et quelles en sont les éventuelles spécificités? Est-ce que la succession est planifiée? Comment le fondateur prévoit-il de s'en occuper? En discute-il avec les membres de la famille? Comment se fait la sélection du ou des successeur(s)? Quel type de relation entretient-t-il avec ces derniers en contexte d'entreprise? Comment se déroule l'intégration du successeur? Quels types de difficultés rencontre-t-il? Quelles sont les réactions des employés ? Ces différentes questions ont servi de trône à notre étude empirique et nous avons tenté de trouver des réponses à ces interrogations. Nous présentons dans la section suivante la démarche méthodologique que nous avons suivie.

\section{La méthodologie de la recherche}

Notre recherche ayant pour but de mieux comprendre, dans une approche exploratoire, le déroulement de la succession en entreprise familiale, la méthode qualitative nous a semblé la plus appropriée pour répondre à cet objectif. La stratégie pour laquelle nous avons optée est celle de l'étude de cas. En effet, ayant comme objectif la compréhension d'un phénomène à l'aide de l'étude intensive d'une situation ou d'un fait spécifique (Yin, 1990), elle nous a semblé bien adaptée à notre questionnement. L'objectif de l'étude de cas ne se réduit pas à l'exploration d'un phénomène peu connu, il peut être de tester ou de générer une théorie ou encore de fournir une représentation intelligible, ce qui s'inscrit dans notre ligne de pensée.

Les entreprises familiales de notre échantillon oeuvrent dans différents secteurs, elles génèrent un chiffre d'affaires annuel avoisinant les 36 millions de dinars ${ }^{3}$ et elles emploient un millier de personnes. Aussi et surtout, le ou les successeur(s) doivent être déjà en place dans l'entreprise familiale. La grande difficulté pour le choix des entreprises a consisté dans le fait qu'en Tunisie, il n'existe pas de statut juridique reconnu de l'entreprise familiale. Pour cette raison, nous avons eu recours à différentes sources dont les annuaires d'entreprises et la liste du classement des entreprises tunisiennes ${ }^{4}$. La première source nous a aidé à sélectionner les entreprises ayant la mention « et fils » dans leur raison sociale et la seconde nous a permis de repérer celles où le nom du dirigeant figurait dans la raison sociale. Parmi les soixante entreprises sélectionnées de cette manière et contactées ensuite par téléphone et à qui nous avons envoyé notre projet de recherche, seulement six entreprises correspondant aux critères évoqués précédemment ont accepté de participer à notre étude.

\footnotetext{
${ }^{3}$ Approximativement 1 euro $=1,5$ dinars tunisiens

${ }^{4}$ Afrique Economie, ${ }^{\circ} 320$, Avril 2003.
} 
L'échantillon

\begin{tabular}{|l|l|c|c|c|c|c|}
\cline { 2 - 7 } \multicolumn{1}{c|}{} & $\begin{array}{c}\text { Secteur } \\
\text { d'activité }\end{array}$ & $\begin{array}{c}\text { Chiffre } \\
\text { d'affaires }\end{array}$ & Employés & Génération & $\begin{array}{c}\text { Successeurs } \\
\text { identifiés }^{7}\end{array}$ & $\begin{array}{c}\text { Etape } \\
\text { approximative } \\
\text { du processus }\end{array}$ \\
\hline E. 1 & Immobilier & $3-5$ & 80 & 2 & 3 & désengagement \\
\hline E. 2 & Emballage & $11-13$ & 600 & 2 & 2 & intégration \\
\hline E. 3 & $\begin{array}{l}\text { Industrie } \\
\text { électrique }\end{array}$ & $7-9$ & 120 & 2 & 1 & intégration \\
\hline E. 4 & Verrerie & $3-5$ & 90 & 2 & 1 & Règne- conjoint \\
\hline E. 5 & $\begin{array}{l}\text { Agro- } \\
\text { alimentaire }\end{array}$ & $3-5$ & 110 & 2 & 2 & Règne- conjoint \\
\hline E. 6 & $\begin{array}{l}\text { Commerce } \\
\text { international }\end{array}$ & $3-5$ & 10 & 2 & 1 & Règne- conjoint \\
\hline
\end{tabular}

Les données ont été collectées par des entretiens semi directifs. Les entretiens ont été réalisés avec deux guides d'entretien différents : le premier destiné au prédécesseur et le second à son (ou ses) successeur (s). Concernant le prédécesseur, il s'agissait d'abord d'avoir une idée sur l'historique de l'entreprise, le lien du prédécesseur avec l'entreprise familiale et la planification de la succession. Ensuite, nous avons voulu connaître la relation qu'entretenait le prédécesseur avec son successeur au sein de l'entreprise familiale, cherché à identifier dans quelle étape du processus l'entreprise se trouvait et enfin repérer les facteurs qui pouvaient empêcher le prédécesseur de passer définitivement le flambeau et ceux liés à l'avenir de l'entreprise après son départ. Pour le successeur, il a été plutôt question de savoir comment s'est déroulée son intégration au sein de l'entreprise familiale, les difficultés qu'il a rencontrées, les résistances qu'il a perçues ainsi que sa relation avec le prédécesseur et les employés. Les données ont été recueillies en face à face, la durée moyenne de chaque entretien a été d'une heure et demie et tous ont été enregistrés sur microcassettes. En plus des guides d'entretiens, à la fin de chaque entrevue, nous avons donné aux répondants un questionnaire à remplir, construit à partir du modèle de Geert Hofstede (1984) «Values Survey Module» à travers lequel nous avons voulu connaître les valeurs que chacun des deux acteurs privilégiaient.

La méthode d'analyse qui nous a semblé la plus adéquate pour le traitement des données collectées est celle de l'analyse de contenu. En effet, cette méthode repose sur le postulat que la répétition d'éléments de discours (mots, expressions ou significations similaires) révèle les centres d'intérêt et les préoccupations des acteurs et elle a pour objectif l'analyse du contenu manifeste d'une communication (Thiétart, 1999). Cette méthode nous a permis de nous

\footnotetext{
${ }^{5}$ En millions de dinars

${ }^{6}$ Présentes dans l'entreprise lors de notre rencontre

${ }^{7}$ Travaillant dans l'entreprise lors de notre rencontre
} 
intéresser plus au sens des données et aux interprétations des deux principaux acteurs de la succession. Toutefois, cette analyse de données pouvant être quantitative ou qualitative, nous avons adopté la deuxième démarche dont l'objet est d'apprécier l'importance des thèmes dans le discours des acteurs plutôt que de chercher à les mesurer (Thiétart, 1999). Nous présentons et discutons les résultats de notre étude dans la troisième section de notre travail.

\section{Résultats et discussion}

L'élaboration de l'historique et de l'évolution des entreprises de notre échantillon nous a permis de faire une analyse plus précise à travers les thèmes que nous avons préalablement élaborés et ensuite revus après une première lecture des résultats. Nous nous sommes intéressés principalement aux quatre thèmes suivants : la planification, le processus de succession, la relation prédécesseur/successeur et les facteurs de succès ou d'échec de la succession. Parmi ces thèmes, certains sont liés à l'entreprise familiale en tant qu'entité et d'autres aux acteurs de l'entreprise familiale, plus précisément le prédécesseur et son ou ses successeurs. Bien évidemment tous ces thèmes sont en rapport avec la succession qui se situe au cœur de notre recherche. Le premier thème est celui de la planification. En effet, les recherches effectuées sur la succession en entreprise familiale démontrent que l'absence de planification peut conduire à des difficultés voire à l'échec de la transmission ( Morris et al., 1997; Ambrose, 1983; Fiegener et al., 1996; Handler et Kram, 1988).

\subsection{La planification de la succession}

$\mathrm{Au}$ moment de la création de leur entreprise, aucun des fondateurs des six entreprises rencontrées n'a planifié ou n'avait en tête l'idée de transmettre son entreprise à un membre de sa famille. Cependant, une fois qu'au moins un des enfants a rejoint l'entreprise, l'idée de transmission est plus présente. Mais d'abord, pour quelles raisons les enfants rejoignent-ils l'entreprise ? Pour l'entreprise (1), le fils aîné du fondateur a dû rejoindre l'entreprise de son père car ce dernier, ne sachant ni lire ni écrire, a ressenti le besoin à un certain moment qu'une personne de confiance le rejoigne pour s'occuper de tout ce qui est administratif. Le fils aîné a ainsi fait ses débuts et a commencé à occuper différentes autres fonctions. Les deux autres fils du fondateur ont rejoint l'entreprise lorsque cette dernière a connu une période de forte croissance entre 1982 et 1985, une phase assez critique pour l'entreprise où l'entrée des deux autres fils est apparue plus que souhaitable. En ce qui concerne l'entreprise (2), la fille aînée du fondateur, après avoir terminé ses études en sciences juridiques, ne s'est pas sentie obligée d'intégrer l'entreprise familiale mais elle savait que son père avait besoin d'elle. 
La situation de l'entreprise (4) est un autre cas de figure car, après quelques «stages » effectués dans l'entreprise pendant les vacances d'été, le fils du fondateur très intéressé par le travail de son père, a demandé à venir travailler avec lui dans le but justement de prendre plus tard la relève. Dans le cas de l'entreprise (5), le fils aîné du fondateur travaillait pendant les vacances dans l'entreprise sans accorder vraiment d'importance à ce qui s'y passait. Ce n'est qu'après avoir débuté ses études universitaires en gestion que le fils du fondateur a commencé à s'intéresser véritablement à ce qu'il faisait au sein de l'entreprise. Le fondateur remarquant que son fils devenait de plus en plus concerné par le travail en essayant par lui-même de trouver des solutions aux problèmes que l'entreprise rencontrait, lui a demandé d'intégrer « officiellement » l'entreprise. Enfin, le successeur de l'entreprise (6) après avoir terminé ses études, a travaillé pendant deux ans dans une entreprise privée. Jugeant qu'on ne lui attribuait pas suffisamment de responsabilités en rapport avec ses qualifications, le successeur a quitté l'entreprise où il travaillait pour intégrer l'entreprise de son père rejoignant par ailleurs le souhait de ce dernier. Ce que nous pouvons remarquer est que l'intégration des successeurs dans l'entreprise, s'est faite le plus souvent suite à des évènements bien précis. Concernant la question précise de la planification de la relève, les réponses apportées par les prédécesseurs sont assez partagées.

\begin{tabular}{|c|c|}
\hline & Les réponses relatives à la planification de la relève ${ }^{8}$ \\
\hline $\begin{array}{l}\text { Prédécesseur } \\
\text { (2) }\end{array}$ & $\begin{array}{l}\text { «...je n'y pense pas vraiment en ce moment.. de toutes les façons ils sont } \\
\text { pour l'instant avec moi dans l'entreprise et c'est déjà ça...on ne parle pas } \\
\text { de la relève, c'est encore tôt...ils ne sont pas encore prêts à tout gérer ». }\end{array}$ \\
\hline $\begin{array}{l}\text { Prédécesseur } \\
\text { (3) }\end{array}$ & $\begin{array}{l}\text { « plus tard...on ne peut pas penser à la relève avant de s'assurer que la } \\
\text { personne qui lui est destinée est parfaitement capable de le faire ». }\end{array}$ \\
\hline $\begin{array}{l}\text { Prédécesseur } \\
\text { (4) }\end{array}$ & $\begin{array}{l}\text { "la prochaine étape sera ma retraite.. il ne m'a peut être pas laissé le } \\
\text { temps de penser à la planification de la relève, c'est lui qui s'est proposé.. } \\
\text { mais je le lui aurais de toute façon demandé ». }\end{array}$ \\
\hline $\begin{array}{l}\text { Prédécesseur } \\
\text { (5) }\end{array}$ & $\begin{array}{l}\text { « c'est l'avenir de toute l'entreprise qui est en jeu et par conséquent celui } \\
\text { de notre famille aussi...le moment venu on discutera de ça ». }\end{array}$ \\
\hline $\begin{array}{l}\text { Prédécesseur } \\
\text { (6) }\end{array}$ & « l'objet de son intégration était d'assurer la continuation de l'entreprise». \\
\hline
\end{tabular}

En résumé, l'expérience des six entreprises rencontrées quant à la planification de la succession ne contredit pas ce que nous avons trouvé dans la littérature concernant ce sujet. Les prédécesseurs n'accordent pas assez de temps pour réfléchir à la relève et à sa planification (Handler et Kram, 1988 ; Hugron, 1993 ; Cadieux, Lorrain et Hugron, 2000). En effet, en s'occupant des activités quotidiennes de l'entreprise, les fondateurs n'ont pas

\footnotetext{
${ }^{8}$ Le prédécesseur (1) n’a pas donné une réponse claire concernant la planification de la relève.
} 
suffisamment de temps pour réfléchir à la planification de la relève. Il est vrai que les prédécesseurs rencontrés commencent tout juste à penser à la relève lorsque leurs enfants rejoignent l'entreprise, mais ce n'est pas pour autant qu'ils concrétisent leur pensée.

\subsection{L'analyse du processus de succession}

Il faut souligner que pour les six entreprises de notre échantillon il ne leur a pas été toujours évident de nous répondre avec précision sur le passage des étapes ainsi que sur la durée de chacune d'entre elles. Dans les six cas étudiés, chacun des successeurs a commencé par se rendre de temps en temps dans l'entreprise dirigée par leur père. Toutefois, ils ne portaient pas tous, à ce moment, le même degré d'intérêt à l'égard de l'affaire familiale.

Si on se réfère au déroulement de la première phase du processus de succession telle que décrite par Hugron et Dumas (1993) et Handler (1994), l'intention de continuité devrait déjà exister durant cette période. Ce n'est pas le cas pour les entreprises que nous avons rencontrées. Le processus de succession n'est enclenché qu'à partir de la deuxième étape, la première se traduisant par des coups de main effectués de temps en temps par les enfants des fondateurs, pas spécialement considérés, durant cette période, comme des successeurs possibles. Une fois les enfants du dirigeant en place, nous pouvons alors commencer à parler d'intention de continuité et de successeurs «potentiels ». Le choix du successeur fait partie intégrante de cette deuxième étape du processus où la situation devient plus claire. En effet, le successeur doit être officiellement nommé ou présenté en tant que tel, c'est le début du règneconjoint où l'intention de continuité se confirme. Le fondateur de l'entreprise (3) se trouvant actuellement au niveau de cette troisième étape nous dit par exemple « nous prenons la plupart des décisions ensemble, pas toutes mais la majorité ». Il nous informe toutefois qu'ils ont eu à un certain moment, lui et son fils, quelques problèmes au niveau du partage des responsabilités «...on s'est retrouvé à un certain moment en train d'oublier des tâches car chacun de nous pensait que l'autre s'en occuperait...il a fallu s'organiser et préciser les fonctions de chacun $»$.

Il est à souligner qu'à aucun moment la notion du pouvoir n'a été réellement abordée. Ceci nous amène à évoquer la quatrième et dernière étape du processus de succession. Dans notre échantillon, l'entreprise (1) est la seule à avoir dépassé cette phase de désengagement, l'un des trois successeurs nous parle de cette période en disant «mon père, de par l'âge et la taille de l'entreprise qui évoluait et dont l'activité se diversifiait, n'avait plus la capacité de tout contrôler par lui-même...il a mis beaucoup de temps pour l'accepter et décider enfin de déléguer ». Le prédécesseur a en effet montré une certaine résistance face à l'obligation de 
déléguer et son fils aîné en a d'ailleurs beaucoup souffert « mon frère aîné a dû se battre et a même été constamment frustré parce que mon père n'a pas toujours pu déléguer », nous précise le cadet. Ses trois fils étaient déjà dans l'entreprise et ce n'est que quelques années plus tard que le père a enfin pris la décision de se retirer. Il a attribué la gérance totale de l'entreprise à son fils aîné car il tenait absolument à préserver la hiérarchie familiale et à la transposer au niveau de la structure de l'entreprise. Au moment de notre rencontre, il y a une sorte de règne conjoint entre les trois fils du fondateur, chacun est responsable d'une activité. Toutefois, le pouvoir est détenu par le frère aîné. Nous pouvons noter que tant que le prédécesseur est présent dans l'entreprise, il détiendra toujours, à lui seul, le pouvoir. Ceci est d'autant plus vrai même à l'approche de son retrait. Le successeur se verra transmettre progressivement des responsabilités et un savoir-faire mais pas le pouvoir.

\subsection{La relation prédécesseur/successeur}

A travers l'analyse du processus de succession dans le cas des entreprises de notre échantillon, nous avons pu noter une évolution dans les rôles respectifs du prédécesseur et du successeur mais pas tout à fait dans le sens de celle décrite par Hugron et Dumas (1993) et Handler (1994). En effet, une fois dans l'entreprise, les successeurs se voient attribuer progressivement de nouvelles fonctions et responsabilités au fur et à mesure qu'ils avancent dans le processus de succession. Toutefois, cela semble dépendre de leurs capacités d'apprentissage, de leur niveau d'engagement et de la confiance qu'ils suscitent chez leurs prédécesseurs. Les successeurs des entreprises de notre échantillon ont connu des évolutions au niveau des responsabilités et ont tenu, en fonction de l'étape du processus par laquelle ils passaient, le rôle d'assistant ou celui de gestionnaire. Toutefois, aucun d'eux (notamment les successeurs de l'entreprise (1) qui ont dépassé la dernière phase du processus) n'a tenu le rôle de dirigeant même au niveau de la dernière étape. En effet, le fils aîné du fondateur de l'entreprise (1) n'a occupé le rôle de dirigeant que lorsque son père s'est définitivement retiré de l'entreprise et lui a attribué par conséquent ce rôle. Au niveau de la troisième étape du processus de succession, les successeurs participent à la prise de décision mais ils ne sont pas sur un pied d'égalité avec les fondateurs, ces derniers gardant toujours leurs prérogatives. Le rôle du prédécesseur durant cette étape ne se limite pas à celui de superviseur, il est toujours le dirigeant de l'entreprise et son successeur se contente de prendre des décisions avec lui. La phase de désengagement est une sorte de continuation de l'étape qui la précède, le successeur participe davantage à la prise de décisions mais le prédécesseur garde toujours la même place. 
La nature de la relation qui existe entre le prédécesseur et le successeur durant le processus de succession semble passer par trois phases. D'abord, lorsque le successeur intègre l'entreprise de son père, tous les deux se trouvent dans une position délicate, le fils travaillant pour son père et ce dernier faisant travailler son fils, ils ne savent pas quelle attitude adopter. De ce fait, des tensions peuvent se créer entre les deux acteurs, l'enfant pouvant avoir par exemple de nouvelles idées pour améliorer la gestion existante et le père pensant que sa manière de gérer ne peut être que meilleure. Ensuite, ils commencent à apprendre à travailler ensemble, ils communiquent plus et il y a un début de complicité qui se précise à l'étape du règne conjoint lorsque le prédécesseur et le successeur collaborent ensemble; à ce moment, la relation devient plus détendue et les deux acteurs entretiennent une relation de complicité et de confiance. Enfin, au niveau de la phase de désengagement, la tension est de retour et l'ambiance est de nouveau tendue. En effet, le prédécesseur n'arrive plus comme c'est le cas pour l'entreprise (1) à tout contrôler. Il joue alors la carte de la contradiction systématique afin de retarder son retrait définitif et l'acte de transfert de direction au successeur.

Il est intéressant à ce niveau d'évoquer les résistances que peuvent montrer les prédécesseurs à l'approche de leur retrait définitif des activités de l'entreprise. Dans la littérature, Handler et Kram (1988) ont identifié quatre grands groupes de facteurs de résistance : individuels, interpersonnels, organisationnels et environnementaux. " Selon les auteurs, le premier groupe de facteurs de résistance est directement lié au fondateur tandis que le second groupe relève de la résistance provenant de la relation qui existe entre le fondateur et le successeur et aussi avec les membres de la famille. Le troisième groupe de facteurs quant à lui, inclut toute résistance provenant de l'environnement interne de l'entreprise. Enfin, celles provenant de l'environnement externe se situent au niveau du quatrième groupe de facteurs » (Cadieux, 1999, p.126). Dans notre échantillon, l'entreprise (1) qui est la seule à avoir dépassé l'étape de désengagement, ne fait pas apparaître ces types de facteurs de résistance tels que décrits par Handler et Kram (1988). Toutefois, nous pouvons souligner que le prédécesseur de l'entreprise (1) a longtemps résisté parce qu'il sentait qu'il était encore capable de tout gérer et ne s'est retiré définitivement que suite à des problèmes de santé. Nous avons posé la question aux successeurs des autres entreprises de notre échantillon pour connaître les éventuelles résistances des prédécesseurs, leurs réponses sont présentées dans le tableau ci-dessous. 


\begin{tabular}{|l|l|}
\cline { 2 - 3 } \multicolumn{1}{c|}{} & \multicolumn{1}{c|}{ Les réponses relatives aux résistances des prédécesseurs } \\
\hline Successeur (2) & «je ne sais pas comment ça se passera...on n'en n'est pas là ». \\
\hline Successeur (3) & $\begin{array}{l}\text { «je pense qu'il va avoir du mal à tout déléguer ...il s'occupe de tout, il n'a } \\
\text { pas vraiment le temps d'avoir d'autres centres intérêts ». }\end{array}$ \\
\hline Successeur (4) & $\begin{array}{l}\text { « ..au début c'est normal que mon père, ne connaissant pas encore mes } \\
\text { aptitudes ne me fasse pas entièrement confiance...c'est même légitime ». }\end{array}$ \\
\hline Successeur (5) & « ..Jusqu'à aujourd'hui, je n'ai pas vraiment senti de résistances ». \\
\hline Successeur (6) & $\begin{array}{l}\text { «..non pas vraiment, il m'a déjà délégué pas mal de responsabilités, il } \\
\text { faut dire qu'il a d'autres occupations depuis quelques temps ». }\end{array}$ \\
\hline
\end{tabular}

Nous pouvons remarquer que dans notre échantillon, les résistances qui existent ou qui pourraient apparaître peuvent provenir de la bonne santé physique et intellectuelle des prédécesseurs et de leur manque de centres d'intérêts en dehors de l'entreprise, ce qui se situe au niveau individuel des facteurs de résistances élaborés par Handler et Kram (1988).

\subsection{Les facteurs de succès ou d'échec de la succession}

Dans la littérature étudiée sur les facteurs de succès de la succession, certains auteurs font allusion à la planification et à la relation prédécesseur/successeur, d'autres évoquent la confiance, la communication et le partage des valeurs et bien d'autres facteurs encore. Nous avons retrouvé cette diversité des facteurs au niveau des réponses des personnes rencontrées.

\begin{tabular}{|c|c|}
\hline & Les réponses relatives aux facteurs de succès ou d'échec ${ }^{9}$ \\
\hline Successeur (1) ${ }^{10}$ & $\begin{array}{l}\text { "pour réussir la succession, il y a toute une culture à développer } \\
\text { indépendamment de la valeur financière, sociale et commerciale de } \\
\text { l'entreprise...pour une gestion saine et efficace, il y a des principes de } \\
\text { base qui doivent être développés par le fondateur lui-même qu'il } \\
\text { transmettra que ce soit à ses enfants ou à des personnes étrangères ». }\end{array}$ \\
\hline Successeur (3) & $\begin{array}{l}\text { « je pense que l'essentiel est de définir...ou peut être délimiter je ne sais } \\
\text { pas les responsabilités de chacun ». }\end{array}$ \\
\hline Fondateur (2) & $\begin{array}{l}\text { " travailler, travailler et encore travailler, il faut apprendre à prendre le } \\
\text { temps d'apprendre et tout ira pour le mieux ». }\end{array}$ \\
\hline Fondateur (4) & «l'échec pourrait arriver quand on ne réfléchit pas à long terme». \\
\hline Fondateur (6) & $\begin{array}{l}\text { «le succès dépend de la culture et la qualité de la transmission...si le père } \\
\text { a cette mentalité de vouloir toujours tout commander et bien ça échoue } \\
\text { parce que l'enfant ne veut pas se sentir écrasé et humilié, il veut trouver } \\
\text { une ambiance d'épanouissement. En revanche, si l'enfant sent qu'on } \\
\text { apprécie ses initiatives et qu'il est écouté, à ce moment cette culture de } \\
\text { souplesse, d'accompagnement et de confiance permettra le succès de la } \\
\text { transmission ». }\end{array}$ \\
\hline
\end{tabular}

\footnotetext{
${ }^{9}$ Les personnes rencontrées mais qui ne figurent pas dans ce tableau n'ont pas donné de réponse à cette question

${ }^{10}$ Le cadet des trois frères
} 
Nous pouvons noter que les personnes rencontrées font souvent allusion à l'importance de la culture et des valeurs. Nous leur avons tous administré à la fin de l'entretien un questionnaire élaboré à l'aide du modèle de Geert Hofstede (1984) «Values Survey Module » à travers lequel nous avons voulu connaître les valeurs que chacun des deux acteurs privilégiait, le but étant de comparer les valeurs des prédécesseurs et celles des successeurs en Tunisie.

\begin{tabular}{|c|c|c|}
\hline & Eléments extrêmement ou très importants & $\begin{array}{l}\text { Eléments peu ou } \\
\text { pas importants }\end{array}$ \\
\hline Prédécesseurs & $\begin{array}{l}\text { apporter une contribution réelle au succès de leur } \\
\text { entreprise ; avoir un travail non routinier; avoir à } \\
\text { relever des défis professionnels à travers lesquels ils } \\
\text { évoluent ; travailler avec des personnes qui acceptent la } \\
\text { coopération; avoir peu de stress dans le travail ; assurer } \\
\text { à eux et à leur famille de bonnes conditions de vie. }\end{array}$ & $\begin{array}{l}\text { - travailler dans } \\
\text { une prestigieuse et } \\
\text { performante } \\
\text { entreprise. }\end{array}$ \\
\hline Successeurs & $\begin{array}{l}\text { avoir de bonnes relations avec leur supérieur } \\
\text { hiérarchique ; assurer à eux et à leur famille de bonnes } \\
\text { conditions de vie ; apporter une contribution réelle au } \\
\text { succès de leur entreprise ; avoir l'opportunité d'évoluer } \\
\text { dans leur travail ; avoir une sécurité d'embauche. }\end{array}$ & $\begin{array}{l}\text { - avoir peu ou pas } \\
\text { de stress dans le } \\
\text { travail. }\end{array}$ \\
\hline
\end{tabular}

Les prédécesseurs et les successeurs ont à ce niveau plusieurs points en commun. En effet, ils accordent une grande importance au fait de contribuer réellement à la réussite de leur entreprise et d'assurer à leur famille le bien être. En revanche, tandis que les prédécesseurs préfèrent éviter le stress dans le travail, les successeurs n'ont pas vraiment de problèmes à ce niveau. Cela pourrait s'expliquer par la différence d'âge : nous ne savons pas en effet ce que pourraient nous répondre ces mêmes successeurs dans vingt ou trente ans. Nous pouvons en conclusion noter l'importance qu'accordent, aussi bien les fondateurs que les successeurs, à l'entreprise et à la famille.

\section{Conclusion}

L'entreprise familiale est un corps vivant qui est en évolution constante et qui se trouve à tout instant confrontée aux changements affectant son environnement. Partant de l'actualité et du grand défi que pose la question de la transmission des entreprises familiales, cette étude s'est proposée de décrire le processus de succession dans les entreprises familiales en mettant l'accent sur le comportement du prédécesseur et celui du successeur durant cette période, ce point étant peu développé en contexte de succession.

D’une façon complémentaire à la majorité des autres recherches qui ont tenté d'analyser la succession dans le contexte européen ou américain (soulignons la quasi-absence de ce type de recherche en Tunisie), nous avons essayé d'avoir une approche globale de ce problème impliquant le recours à des entretiens semi directifs pour comprendre cette relation durant 
toutes les phases de la succession à travers une enquête qui a touché aussi bien les prédécesseurs que les successeurs. Notre étude exploratoire menée auprès de six entreprises familiales tunisiennes nous a permis de clarifier quelques notions peu développées dans des travaux antérieurs. Ainsi, nous avons pu avancer l'idée que la qualité de la relation entre le dirigeant et son successeur est primordiale au bon déroulement du processus de succession et que le succès de ce dernier ne dépend pas uniquement de la volonté du fondateur à lâcher prise mais aussi de la capacité d'apprentissage dont doit faire preuve le successeur. La nature de la relation que les deux acteurs entretiennent dépend de la phase du processus dans laquelle l'entreprise se trouve et des résistances peuvent apparaître bien avant la phase de désengagement du prédécesseur, au niveau de l'intégration du successeur. Aussi, la relation prédécesseur/ successeur passe par trois états : tendue au moment de l'intégration du successeur, une certaine complicité se développe par la suite au niveau du règne conjoint et enfin leur relation est de nouveau tendue au moment du désengagement du prédécesseur. Ce dernier montre une certaine résistance face à son désengagement et n'accepte pas de déléguer tant qu'il se sent capable de tout gérer par lui-même. Ayant consacré tout son temps et son énergie à l'entreprise, le prédécesseur a du mal à prendre la décision de se retirer, la peur d'une perte d'identité ainsi qu'un très grand vide le hantent. En effet, tant que le fondateur est présent dans l'entreprise, même à l'approche de son retrait, il détient à lui seul le pouvoir. Tout au long du processus de succession, il y a un transfert de responsabilités, de savoir-faire et de leadership mais pas de pouvoir, ce dernier ne pouvant être partagé. Le successeur devra montrer son engagement envers l'entreprise et susciter un niveau élevé de confiance chez le prédécesseur s'il veut évoluer et passer à des niveaux supérieurs. L'existence d'un lien de parenté facilite la tâche puisqu'ils cherchent tous les deux la réussite de l'entreprise familiale. Allouche et Amann (1998) mentionnent que la confiance est un facteur explicatif de la performance des entreprises familiales.

Enfin, le succès de la succession dans une entreprise familiale est tributaire d'une culture caractérisée par l'esprit d'équipe, la confiance, la souplesse et l'efficacité qui doit être transmise à tous les membres de l'entreprise, autant à ceux qui appartiennent à la famille qu'à ceux qui n'en font pas partie. Cependant, plusieurs questions restent en suspens et appellent à d'autres recherches. En effet, il serait intéressant de mener une étude similaire sur un échantillon plus grand avec des processus de succession père-fils et père-fille de manière à conférer à l'étude un caractère plus généralisable. D'un autre côté, nous ne savons pas comment le successeur se comporte une fois qu'il est seul à bord ? Est-ce qu'il y a une rupture 
ou une continuité dans les modes de gestion? Quelle est la nature de la relation successeur/employés lorsque le prédécesseur se retire définitivement? Quelles en sont les conséquences ? Ceci nous amène à évoquer les limites de notre recherche. D'abord la stratégie de recherche utilisée, celle de l'étude de cas, bien qu'intéressante parce qu'elle nous permet d'étudier le concept en profondeur, ne nous permet pas d'examiner les différents cas de figure, susceptibles de se présenter et par conséquent ne permet pas d'aboutir à une généralisation à l'ensemble des entreprises familiales tunisiennes mais aussi aux entreprises d'autres pays. De plus, s'agissant d'un processus, une méthode d'observation participante aurait pu compléter la méthode que nous avons utilisée. Toutefois, cette approche de recherche est basée sur une longue période, ce qui a limité son emploi dans le cadre de ce travail.

\section{Références}

Allouche J. et Amann B. (1998), La confiance : une explication des performances des entreprises familiales, Economies et Sociétés, Série SG, n8/9, pp.129-154.

Allouche J. et Amann B. (2003), L'actionnaire dirigeant d'entreprise familiale, Revue Française de Gestion, 28, 141, pp.109-130.

Ambrose D. (1983), Transfer of the Family-owned Business, Journal of Small Business Management, vol.21, pp.49-56.

Barach et al. (1988), Entry of the Next Generation: Strategic Challenge for Family Business, Journal of Small Business Management, Avril, pp.49-56.

Barnes L.-B. et Hershon S.-A. (1976), Transferring Power in Family Business, Harvard Business Review, juillet - août, pp.105-114.

Baumert H. (1992), Succession dans la PME familiale : prévoir pour réussir, Les Editions d'organisation.

Bayad M. et Barbot M.-C. (2002), Proposition d'un modèle de succession dans les PME familiales : étude de cas exploratoire de la relation père-fille, $6^{\text {ème }}$ congrès international francophone sur la PME, octobre, HEC-Montréal.

Bruneau J.-P. (2002), Psychanalyse et entreprises : création, développement et transmission des PME, Les Presses du Management. 
Cadieux L. (1999), La succession en entreprise familiale: analyse du processus dans le cas de quatre entreprises manufacturières fondées par des femmes, Mémoire présenté à l'Université du Québec à Trois-Rivières, 187 p.

Cadieux L. et Lorrain J. (2002), Le processus de succession dans les entreprises familiales, $6^{\text {ème }}$ congrès international francophone sur la PME, octobre, HEC, Montréal.

Cadieux L., Hugron P. et Lorrain J. (2000), La succession dans les entreprises familiales : une étude de cas exploratoire faite auprès de quatre PME manufacturières fondées et dirigées par des femmes, $5^{\text {ème }}$ congrès international francophone sur la PME, octobre, Lille.

Carter B. et McGoldrick M. (1988), The Changing Family Life Cycle: A Framework for Family Therapy, Second Edition, Gardness Press, New York.

Catry B. et Buff A. (1996), Le gouvernement de l'entreprise familiale, Publi-Union Edition.

Churchill N.-C. et Hatten K.-J. (1987), Non-market Based Transfers of Wealth and Power: A research Framework for Family Businesses, American Journal of Small Business, 11, 3.

Davis J.-A. et Tagiuri R. (1982), Bivalent Attributes of the Family Firm, Santa Barbara, CA, Owner Managed Business Institute.

Deslauriers J.-P. (1991), Recherche qualitative : guide pratique, McGraw-Hill, Montréal.

Duchéneaut B. (1996), Les dirigeants de PME, Maxima, Laurent du Mesnil Editeur.

Dyer G. et Handler W.-C. (1994), Entrepreneurship and Family Business: Exploring the Connections, Entrepreneurship Theory \& Practice, 19, 1, pp.71-83.

Fiegener M.-K. (1996), A Comparison of Successor Development in Family and Non-family Businesses, Family Business Review, n9, pp.313-329.

Fiegener M.-K. et al. (1996), Passing on Strategic Vision, Journal of Small Business Management, 34, 3, pp.15-26.

Gallo M.-A. et Estapé M.-J. (1992), A Family Business Among the Top 1000 Spanish Companies, IESE Research Paper $n^{\circ} 231$. 
Gersick H.-E., Davis J.-A., McCollom H.-M. et Lansberg S.-I. (1997), Generation to Generation, Life Cycles of the Family Business, Harvard Business School Press,

Ghertman M. (1996), Management stratégique de l'entreprise, PUF, Paris.

Haddadj S. et D’Andria A. (1998), Transmissions internes et transmissions externes dans les PME françaises: existe-t-il des différences de changements stratégiques et d'orientation stratégiques?, Revue Internationale des PME, 11, 4, pp.45-66.

Haddadj S. et D'Andria A. (2001), Mesure des phénomènes qui agissent sur la transmission des PME familiales, Revue Française de Gestion, n¹32, jav-fév.

Handler W.-C. (1989), Methodological Issues and Considerations in Studying Family Businesses, Family Business Review, n², pp.257-276.

Handler W.-C. (1990), Succession in Family Firms: A Mutual Role Adjustment Between Entrepreneur and Next-generation Family Members, Entrepreneurship Theory and Practice, 15,1, pp.37-51.

Handler W.-C. et Kram K.-E. (1988), Succession in Family Firms : The Problem of Resistance, Family Business Review, 1, 4, pp.361-381.

Hofstede G. (1984), Culture's consequences: international differences in work-related values, London, Sage.

Hollander B. et Elman N. (1988), Family-owned Businesses : An Emerging Field of Inquiry, Family Business Review, n ${ }^{\circ}$, pp.145-164.

Hugron P. (1993), L'entrepreneur et la succession d'entreprise : analyse de 12 processus, Cahier de recherche CREF 93-03, Ecole des Hautes Etudes Commerciales.

Hugron P. et Boiteux S. (1998), La PME familiale mondiale : conséquence sur la relève, Actes du $4^{\text {ème }}$ congrès international francophone sur la PME.

Hugron P. et Dumas C. (1993), Modélisation du processus de succession des entreprises familiales québécoises, Cahier de Recherche nªREF-93-07, HEC.

Kets de Vries M.-F.-R. (1988), Succession du PDG : L'ombre au tableau, Harvard L'Expansion, $\mathrm{n}^{\circ} 50$, Automne, pp.99-104. 
Lacourse M.-T. et coll. (1994), Famille et société, McGraw-Hill, Montréal.

Meister V. (1999), Etude organisationnelle d'une PME familiale, sensibilisation et changement, Mémoire pour le Diplôme Postgrade en Informatique et Organisation, HEC, Université de Lausanne.

Morris M.-H. et al. (1997), Correlates of Success in Family Business in Transition, Journal of Business Venturing, 12, 5, pp.385-401.

Pailot P. (1998), Propositions théoriques et épistémologiques pour une méthodologie d'analyse des freins "psychologiques" des dirigeants de PME lors des transmissions d'entreprise, CIFPME, IAE de Metz, octobre.

Rousillon S. (1998), Approche psychologique de la préparation des dirigeants, in Bournois F. et Rousillon S. (1998), Préparer les dirigeants de demain, Editions d'organisation.

Stravou Z.-T. et Swiercz P.-M. (1998), Securing the Future of the Family Enterprise: A Model of Offspring Intentions to Join the Business, Entrepreneurship Theory and Practice, 23, 2, pp.19-39.

Tchankam J.-P. (1998), Performances comparées des entreprises publiques et privées au Cameroun, thèse de doctorat en sciences de gestion, Université Montesquieu Bordeaux, Prix de l'Académie Nationale des Sciences, Belles -Lettres et Arts de Bordeaux.

Thiétart R.-A. et coll. (1999), Méthodes de recherches en management, Paris, Dunod.

Wacheux F. (1996), Méthodes qualitatives et recherche en gestion, Economica.

Yin R.-K. (1990), Case Study Research : Design and Methods, Sage Publications Inc. 